\title{
A contrast source method for nonlinear acoustic wave fields in media with spatially inhomogeneous attenuation
}

\author{
L. Demi ${ }^{a}$ and K. W. A. van Dongen \\ Laboratory of Acoustical Imaging and Sound Control, Faculty of Applied Sciences, Delft University \\ of Technology, Lorentzweg 1, 2628 CJ Delft, The Netherlands \\ M. D. Verweij \\ Laboratory of Electromagnetic Research, Faculty of Electrical Engineering, Mathematics and Computer \\ Science, Delft University of Technology, Mekelweg 4, 2628 CD Delft, The Netherlands
}

(Received 24 June 2010; revised 21 December 2010; accepted 26 December 2010)

\begin{abstract}
Experimental data reveals that attenuation is an important phenomenon in medical ultrasound. Attenuation is particularly important for medical applications based on nonlinear acoustics, since higher harmonics experience higher attenuation than the fundamental. Here, a method is presented to accurately solve the wave equation for nonlinear acoustic media with spatially inhomogeneous attenuation. Losses are modeled by a spatially dependent compliance relaxation function, which is included in the Westervelt equation. Introduction of absorption in the form of a causal relaxation function automatically results in the appearance of dispersion. The appearance of inhomogeneities implies the presence of a spatially inhomogeneous contrast source in the presented full-wave method leading to inclusion of forward and backward scattering. The contrast source problem is solved iteratively using a Neumann scheme, similar to the iterative nonlinear contrast source (INCS) method. The presented method is directionally independent and capable of dealing with weakly to moderately nonlinear, large scale, three-dimensional wave fields occurring in diagnostic ultrasound. Convergence of the method has been investigated and results for homogeneous, lossy, linear media show full agreement with the exact results. Moreover, the performance of the method is demonstrated through simulations involving steered and unsteered beams in nonlinear media with spatially homogeneous and inhomogeneous attenuation. (C) 2011 Acoustical Society of America. [DOI: $10.1121 / 1.3543986]$
\end{abstract}

PACS number(s): 43.25.Jh, 43.25.Cb, 43.35.Bf, 43.80.Qf [TDM] Pages: 1221-1230

\section{INTRODUCTION}

Nonlinear acoustics play a key role in the development of new medical diagnostic and therapeutic applications of ultrasound. For example, nonlinear ultrasound is known to significantly improve the quality of echographic images ${ }^{1}$ and the heat deposition during acoustic ablation therapy. ${ }^{2}$ These effects are due to the higher harmonic wave fields that come along with a high amplitude fundamental wave field in case of nonlinear propagation.

Among the peculiarities of the higher harmonic wave fields of an acoustic beam are a reduction of the axial and lateral size of the focal spot, clutter, and side and grating lobes, ${ }^{1}$ as compared to the fundamental wave field. A well known ultrasound imaging modality is tissue harmonic imaging (THI) ${ }^{3,4}$ This technique takes advantage of the mentioned benefits by imaging only the reflection of the second harmonic component. To further exploit the benefits of higher harmonics, researchers currently investigate ultrasound imaging based on the reflection of the third to the fifth harmonic component. In the literature this is referred to as superharmonic imaging (SHI). ${ }^{5,6}$

Experimental data reveal that attenuation or loss is another phenomenon of substantial importance in medical

\footnotetext{
a) Author to whom correspondence should be addressed. Electronic mail: 1.demi@tudelft.nl
}

applications of ultrasound. At ultrasonic frequencies, attenuation in biological tissue is mainly due to absorption, i.e., the irreversible conversion of acoustic energy into heat, and to a lesser extent to scattering of the acoustic energy in arbitrary directions. The attenuation coefficient in biological tissue may usually be described by a frequency power law with a positive exponent. ${ }^{7,8}$ Consequently, the higher harmonics experience a greater attenuation than the fundamental wave field. For a given penetration depth, this effect limits the frequency that can be applied for echographic imaging, and thus the attainable resolution. On the other hand, the same effect increases the deposited heat of an applicator for acoustic ablation. ${ }^{2,9}$

The design and optimization of a new ultrasound modality or device that is based on nonlinear acoustics, e.g., a phased array transducer intended for SHI, requires the ability to accurately simulate and understand the corresponding nonlinear phenomena. A particular challenge is to accurately compute the higher harmonic contents of beams that are steered over large angles and that extend over large, threedimensional spatial domains. The iterative nonlinear contrast source (INCS) method ${ }^{10,11}$ is capable of performing such simulations. This method is based on the full-wave Westervelt equation, ${ }^{12}$ and considers its nonlinear term to describe a distributed contrast source that operates in a linear and homogeneous background medium. The contrast source depends nonlinearly on the total wave field, and the wave 
field generated by the contrast source is the nonlinear part of the total wave field. Starting with the linear wave field generated by the primary source (i.e., the transducer) in the background medium, the contrast source and the total wave field are iteratively updated by way of a Neumann scheme. The result of the first iteration is the quasilinear solution. Next, the solution is iteratively improved toward the full nonlinear wave field. As a rule of thumb, for weak to moderate nonlinearity, $n+1$ iterations suffice for an accurate computation of the $n$th harmonic. The key step in each iteration is a spatiotemporal convolution of the distributed contrast source with the Green's function of the background medium. This is performed with the filtered convolution method, ${ }^{10,13}$ which employs a priori filtering and fast Fourier transform (FFT) techniques to allow for a spatiotemporal grid with only two points per wavelength and per period. Due to the contrast source approach and the applied convolution technique, the INCS method shows no directional dependence.

In addition to nonlinearity, medical applications require the effects of attenuation and dispersion to be included in the simulations of the acoustic wave field. With the INCS method, attenuation has already been taken into account by using a Green's function for a background medium with frequency power law losses. ${ }^{10,14}$ However, this approach cannot deal with situations where the attenuation varies throughout the spatial domain, as commonly occurs in biomedical situations. For the linear case, methods have been applied that take these inhomogeneities into account. ${ }^{15}$ The purpose of this paper is to show how spatially inhomogeneous attenuation may be incorporated in the INCS method.

To deal with the stated problem, the attenuation is modeled via a causal compliance relaxation function that describes how the pressure history up to a particular moment contributes to the cubic dilatation rate at that moment. ${ }^{10,14,16,17}$ Inhomogeneous losses are incorporated in the model of nonlinear acoustics by including a spatially dependent compliance relaxation function in the Westervelt equation. This equation is then considered to describe two distributed contrast sources in a linear, homogeneous, and lossless background medium; ${ }^{18}$ the nonlinear contrast source provides the nonlinear contribution to the wave field, and the attenuation contrast source yields the correction due to the spatially inhomogeneous losses. Finally the resulting contrast source problem is solved for the unknown wave field in an iterative way by using a Neumann scheme and the filtered convolution method. ${ }^{10,13}$

In the literature, several other methods have been described for the incorporation of attenuation in the equations of nonlinear acoustics. One approach includes a series of simple relaxation terms in the KZK equation ${ }^{19}$ or in the Westervelt equation. ${ }^{20}$ Another approach introduces a loss operator in the Burgers equation ${ }^{21}$ or in the Khokhlov-Zabolotskaya-Kuznetsov (KZK) equation. ${ }^{22}$ In the current paper, preference is given to the application of a compliance relaxation function because it allows for a compact way of modeling the frequency power law attenuation shown by many biological tissues, and it automatically provides the associated dispersion. More importantly, by including a spatially dependent relaxation function in Westervelt equation, the resulting full-wave method can cope with attenuation that varies in all three spatial directions, and it yields both the forward and backward scattering generated by this.

In Sec. II, the theory is formulated through the introduction of the basic equations and the spatially dependent compliance relaxation function. The iterative solution method will be presented in Sec. III. In Sec. IV, the convergence of the method is investigated, next numerical results obtained for homogeneous, nonlinear, and lossy media are compared with the results obtained for homogeneous, nonlinear, and lossless media to emphasize how attenuation acts differently with respect to the fundamental component of the wave field and the higher harmonics. Moreover, results obtained for a nonlinear medium with spatially inhomogeneous losses are presented to show the capability of the method to model forward and backward scattering effects. Conclusions of the presented research are given in Sec. V.

\section{THEORY}

\section{A. Extending the wave equation to nonlinear and attenuating media}

In this subsection, the wave equation for a homogeneous, linear, and lossless medium will be extended to deal with nonlinearity, spatially inhomogeneous attenuation, and a combination of both effects. The resulting equations will be cast in a form involving a contrast source.

\section{Linear wave equation}

In a homogeneous, linear, and lossless medium the propagation of the acoustic pressure field $p(x, t)$ is described by the wave equation

$$
\nabla^{2} p(\boldsymbol{x}, t)-\frac{1}{c_{0}^{2}} \partial_{t}^{2} p(\boldsymbol{x}, t)=-S_{\mathrm{pr}}(\boldsymbol{x}, t)
$$

Here, the vector $x$ contains the coordinates of a position in a three-dimensional Cartesian domain $\mathcal{D}$, and $t$ is the time coordinate. The symbol $\nabla^{2}=\nabla \cdot \nabla$ indicates the Laplace operator, and $\partial t$ indicates differentiation with respect to time. The behavior of the homogeneous, linear, and lossless acoustic medium is described via the ambient speed of sound $c_{0}$ and the ambient volume density of mass $\rho_{0}$. The acoustic wave field is generated by the primary source $S_{\mathrm{pr}}(x, t)$. This source depends on the volume density of injection rate source $q(x, t)$ and the volume density of volume force source $\mathbf{f}(x, t)$ according to

$$
S_{\mathrm{pr}}(\boldsymbol{x}, t)=\rho_{0} \partial_{t} q(\boldsymbol{x}, t)-\nabla \cdot \mathbf{f}(\boldsymbol{x}, t) .
$$

Equation (1) yields an accurate description of the acoustic pressure field for small-amplitude disturbances, i.e., when nonlinear effects are negligible.

\section{Inclusion of nonlinearity}

In medical applications where high amplitude wave fields are used, the wave propagation becomes inherently nonlinear and Eq. (1) must be extended to cope with this fact. A 
suitable wave equation for a homogeneous, nonlinear, and lossless acoustic medium is the Westervelt equation ${ }^{12}$

$$
\nabla^{2} p(\boldsymbol{x}, t)-\frac{1}{c_{0}^{2}} \partial_{t}^{2} p(\boldsymbol{x}, t)=-S_{\mathrm{pr}}(\boldsymbol{x}, t)-\frac{\beta}{\rho_{0} c_{0}^{4}} \partial_{t}^{2} p^{2}(\boldsymbol{x}, t),
$$

in which the nonlinear behavior of the acoustic medium is described via the coefficient of nonlinearity $\beta$. This equation is exact up till the terms of second order in the acoustic disturbance quantities, takes into account the global nonlinear effects, and neglects local nonlinear effects. ${ }^{23}$

In medical diagnostic applications, the effect of the nonlinear term on the total wave field remains small, but noticeable. In that case it makes sense to consider this term to describe a distributed pressure dependent secondary or contrast source

$$
S_{\mathrm{nl}}[p(\boldsymbol{x}, t)]=\frac{\beta}{\rho_{0} c_{0}^{4}} \partial_{t}^{2} p^{2}(\boldsymbol{x}, t) .
$$

This is referred to as the nonlinear contrast source. ${ }^{10,11,13}$ The left-hand side of Eq. (3) represents the wave operator corresponding to a homogeneous, linear, and lossless medium. In this linear background medium, the nonlinear contrast source $S_{\mathrm{nl}}$ generates an additional field that is equal to the nonlinear part of the total acoustic field in the nonlinear medium.

\section{Inclusion of attenuation}

In most medical applications of ultrasounds, the medium cannot be considered lossless and attenuation must be accounted for. Usually, the attenuation of the medium depends on the position. To describe pressure fields in a linear medium with spatially inhomogeneous attenuation, a spatially dependent loss mechanism should be incorporated in the wave equation. A pragmatic approach is to start with a known expression for the lossless wave field. This lossless expression is turned into a lossy one by simply adding an imaginary part to the frequency, or by incorporating a real attenuation coefficient $\alpha(\omega)$ in the propagation coefficient $\gamma(\omega)$. This pragmatic approach, however, can hardly be used to model spatially inhomogeneous losses. A more fundamental approach is to extend the equation of deformation and motion, or the wave equation, by adding loss terms with a higher order time derivative, or by introducing relaxation in the medium behavior. Solution of these equations directly provides the lossy wave field. In this paper, a causal relaxation function is employed because it provides a compact way of modeling frequency power law attenuation, it automatically yields the associate dispersion, and it allows for a straightforward extension to spatially inhomogeneous losses.

Using the relaxation function approach, the lossless deformation equation,

$$
\nabla \cdot \mathbf{v}(\boldsymbol{x}, t)+\kappa_{0} \partial_{t} p(\boldsymbol{x}, t)=q(\boldsymbol{x}, t)
$$

may be turned into its lossy counterpart ${ }^{16,17}$

$$
\nabla \cdot \mathbf{v}(\boldsymbol{x}, t)+\kappa_{0} \partial_{t}\left[\chi(\boldsymbol{x}, t) *_{t} p(\boldsymbol{x}, t)\right]=q(\boldsymbol{x}, t) .
$$

Here, $\kappa_{0}$ is the ambient compressibility, $v$ is the particle velocity, and $\chi(x, t)$ is the so-called normalized compliance (or memory) relaxation function. The latter function depends on $x$ to model spatially dependent attenuation. It may be separated into

$$
\chi(\boldsymbol{x}, t)=\delta(t)+A(\boldsymbol{x}, t)
$$

where the Dirac delta function represents the instantaneous medium behavior, and the relaxation function $A(x, t)$ represents the delayed reaction of the medium on events that happened in the past. The latter behavior is associated with the occurrence of losses. Because of its physical role, the relaxation function $A(x, t)$ is real valued. Moreover, it may not predict the future and it may not contribute to the instantaneous medium behavior. This implies that $A(x, t)$ is a causal function that does not include a function $\delta(t)$ or functions with a higher singular behavior at $t=0$. The real-valuedness and causality of $A(x, t)$ imply the real-valuedness and causality of $\chi(t)$.

In the same way, the equation of motion,

$$
\nabla p(\boldsymbol{x}, t)+\rho_{0} \partial_{t} \mathbf{v}(\boldsymbol{x}, t)=\mathbf{f}(\boldsymbol{x}, t)
$$

may be turned into its lossy version by convolving $v$ with a normalized inertia relaxation function $\mu(t) .{ }^{16,17}$ However, it is usually assumed that in biological tissue attenuation is dominant due to relaxation in the compliance, while the inertia shows negligible relaxation effects.

Combination of Eqs. (6) and (8) yields the following wave equation for linear acoustic media with spatially inhomogeneous losses:

$$
\nabla^{2} p(\boldsymbol{x}, t)-\frac{1}{c_{0}^{2}} \partial_{t}^{2}\left[\chi(\boldsymbol{x}, t) *_{t} p(\boldsymbol{x}, t)\right]=-S_{\mathrm{pr}}(\boldsymbol{x}, t),
$$

with $c_{0}=\left(\rho_{0} \kappa_{0}\right)^{-\frac{1}{2}}$. Substitution of Eq. (7) into Eq. (9) allows the latter to be rewritten as

$$
\begin{aligned}
\nabla^{2} p(\boldsymbol{x}, t)-\frac{1}{c_{0}^{2}} \partial_{t}^{2} p & (\boldsymbol{x}, t) \\
& =-S_{\mathrm{pr}}(\boldsymbol{x}, t)+\frac{1}{c_{0}^{2}} \partial_{t}^{2}\left[A(\boldsymbol{x}, t) *_{t} p(\boldsymbol{x}, t)\right]
\end{aligned}
$$

Similar to the nonlinear term in Eq. (3), an attenuation term now appears at the right-hand side of Eq. (10). In medical applications, the effect of the attenuation term on the total wave field usually remains small, and it makes sense to consider it to describe a distributed pressure dependent secondary or contrast source

$$
S_{\text {at }}[p(\boldsymbol{x}, t)]=-\frac{1}{c_{0}^{2}} \partial_{t}^{2}\left[A(\boldsymbol{x}, t) *_{t} p(\boldsymbol{x}, t)\right]
$$

This will be referred to as the attenuation contrast source.

\section{Combination of nonlinearity and attenuation}

The attenuation contrast source in Eq. (11) may be combined with the nonlinear contrast source in Eq. (4) to obtain 
the following wave equation for nonlinear media with spatially inhomogeneous losses

$$
\begin{aligned}
\nabla^{2} p(\boldsymbol{x}, t)-\frac{1}{c_{0}^{2}} & \partial_{t}^{2} p(\boldsymbol{x}, t) \\
& =-S_{\mathrm{pr}}(\boldsymbol{x}, t)-S_{\mathrm{nl}}[p(\boldsymbol{x}, t)]-S_{\mathrm{at}}[p(\boldsymbol{x}, t)] .
\end{aligned}
$$

This may be written as

$$
\nabla^{2} p(\boldsymbol{x}, t)-\frac{1}{c_{0}^{2}} \partial_{t}^{2} p(\boldsymbol{x}, t)=-S_{\mathrm{pr}}(\boldsymbol{x}, t)-S_{\mathrm{cs}}[p(\boldsymbol{x}, t)],
$$

in which the total contrast source term equals

$$
S_{\mathrm{cs}}[p(\boldsymbol{x}, t)]=S_{\mathrm{nl}}[p(\boldsymbol{x}, t)]+S_{\mathrm{at}}[p(\boldsymbol{x}, t)] .
$$

Equations (13) and (14) form an extended version of Westervelt equation, which is solved in Sec. III.

\section{B. Compliance relaxation function}

In this subsection, the general properties of a compliance relaxation function are derived. Moreover, a suitable relaxation function is proposed that yields a frequency power law attenuation.

\section{General properties}

For convenience it is first assumed that the attenuation is spatially independent, i.e., the considered compliance memory function is $\chi(x, t)=\chi(t)$. If in that case, Eqs. (6)-(8) are subjected to a temporal Laplace transformation and the proper substitutions are performed, the following wave equation may be obtained:

$$
\nabla^{2} \hat{p}(\boldsymbol{x}, s)-\frac{s^{2}}{c_{0}^{2}} \hat{\chi}(s) \hat{p}(\boldsymbol{x}, s)=-\hat{S}_{\mathrm{pr}}(\boldsymbol{x}, s),
$$

where the hat indicates that a quantity has been transformed by means of the Laplace transformation with parameter $s$.

At this stage, the Laplace transformation is preferred over the Fourier transformation. This is because Lerch's theorem $^{24-26}$ for the Laplace transformation provides a stronger and simpler way of keeping track of causality than the Paley-Wiener ${ }^{27}$ theorem does for the Fourier transformation. From Lerch's theorem it may be deduced that a sufficient condition for a transform domain function $\hat{f}(s)$ to correspond to a unique, causal time domain function $f(t)$ is that $\hat{f}(s)$ should remain bounded for all real $s \geq s_{0} \geq 0$, where $s_{0}$ may be chosen arbitrary large (but not infinite).

Like any of the transformed quantities, $\hat{\chi}(s)$ is the Laplace transform of a real function $\chi(t)$, so $\hat{\chi}(s)$ must be real for real values of $s$. Moreover, $\hat{\chi}(s)$ is the Laplace transform of a unique, causal function $\chi(t)$. As explained in the previous paragraph, this is certainly satisfied if $\hat{\chi}(s)$ remains bounded on the real axis for $s \geq s_{0} \geq 0$.

Upon solving Eq. (15), the obtained wave field will have a propagation coefficient

$$
\gamma(s)=\frac{s}{c_{0}} \hat{\chi}^{\frac{1}{2}}(s),
$$

in which $c_{0}$ is the wave speed in the lossless medium. In the lossless case there is no dispersion and every part of the wave has the same speed $c_{0}$. This speed also applies to the wave front. The speed of the wave front always follows from the high-frequency behavior of $\gamma$ as $c_{0}=\lim _{|s| \rightarrow \infty} s / \gamma(s)$ for $\operatorname{Re}(s)>0$. In case of losses, $\hat{A}(s)$ will be at most of order $s^{n}$ with $n<0$ since $A(t)$ is less singular at $t=0$ than a delta function. Consequently, $\lim _{|s| \rightarrow \infty} \hat{\chi}(s)=1$ and the wave front will travel with the same speed $c_{0}$ as in the lossless case.

When replacing $s$ by $j \omega$, the propagation coefficient may be written as

$$
\gamma(j \omega)=\alpha(\omega)+j \beta(\omega)
$$

in which $\alpha(\omega)$ is the attenuation coefficient and $\beta(\omega)$ is the phase coefficient. In view of the causality of $\chi(t)$, the real and imaginary parts of $\hat{\chi}(j \omega)$ must now satisfy the KramersKronig relations. ${ }^{28}$ As a consequence of causality, $\alpha(\omega)$ cannot be chosen independent from $\beta(\omega)$, and attenuation and dispersion will be interlinked phenomena.

The above theory is easily extended to the case of inhomogeneous losses by taking a spatially dependent compliance relaxation function $\chi(x, t) .{ }^{17}$ From the above, it is possible to derive the general properties of any causal function $\hat{\chi}(\boldsymbol{x}, s):^{10,14}$

(1) $\hat{\chi}(\boldsymbol{x}, s)$ is real for real values of $s$,

(2) $\hat{\chi}(\boldsymbol{x}, s)$ remains bounded for all $s \geq s_{0} \geq 0$, and

(3) $\lim _{|s| \rightarrow \infty} \hat{\chi}(\boldsymbol{x}, s)=1$ for $\operatorname{Re}(s)>0$.

\section{Compliance relaxation function for frequency power law losses}

A function $\hat{\chi}(s)$ that for $s=j \omega$ provides the power law attenuation coefficient $\alpha(\omega)=\alpha_{1}|\omega|^{b}$ as observed in many measurements, ${ }^{7,8,29}$ is

$$
\hat{\chi}(s)=\left(1+\frac{c_{0} \alpha_{1} s^{b-1}}{\cos (\pi b / 2)}\right)^{2}
$$

with positive real parameters $\alpha_{1}$ and $b$ ( $b$ may not be an odd integer). Some typical values are given in Table I, and more extensive data may be found in the literature. ${ }^{8,29-31}$ The given function yields the same attenuation and phase coefficient as obtained by Szabo. ${ }^{32}$

In the current context, two changes will made to Eq. (18). First, in order to satisfy the last two requirements at the end of Sec. II B 1, a dominator is introduced that prevents

TABLE I. Acoustic medium parameters for water and several human tissues (Ref. 30). The relation between $a$ and $\alpha_{1}$ is $\alpha_{1}=100 \times a(2 \pi)^{-b}$.

\begin{tabular}{lcccc}
\hline \hline Medium & $\begin{array}{c}a \\
{\left[\mathrm{~Np} \mathrm{~cm}^{-1} \mathrm{MHz}^{-b}\right]}\end{array}$ & $\begin{array}{c}b \\
{[-]}\end{array}$ & $\begin{array}{c}c_{0} \\
{\left[\mathrm{~ms}^{-1}\right]}\end{array}$ & $\begin{array}{c}\beta \\
{[-]}\end{array}$ \\
\hline Liver & $5.2 \cdot 10^{-2}$ & 1.05 & 1578 & 4.38 \\
Liver background & 0 & - & 1578 & 4.38 \\
Brain & $6.7 \cdot 10^{-2}$ & 1.3 & 1562 & 4.28 \\
Brain background & 0 & - & 1562 & 4.28 \\
Blood & $1.6 \cdot 10^{-2}$ & 1.21 & 1584 & 4 \\
Blood background & 0 & - & 1584 & 4 \\
Water & $2.5 \cdot 10^{-4}$ & 2 & 1482.3 & 3 \\
Water background & 0 & - & 1482.3 & 3 \\
\hline \hline
\end{tabular}


the function to become infinite for $|s| \rightarrow \infty$. Secondly, the relevant loss coefficients are made spatially dependent. The resulting function $\hat{\chi}(\boldsymbol{x}, s)$ is

$$
\hat{\chi}(\boldsymbol{x}, s)=\left(1+\frac{c_{0} \alpha_{1}(\boldsymbol{x}) s^{b(\boldsymbol{x})-1}}{\cos (\pi b(\boldsymbol{x}) / 2)\left[1+\left(s / s_{1}\right)^{d}\right]}\right)^{2} .
$$

The parameters $\alpha_{1}(x)$ and $b(x)$ represent the spatially dependent attenuative properties of the specific medium. Further, $s_{1}$ is a positive parameter that is larger than the largest angular frequency of interest, and $d$ is a positive integer parameter that satisfies $d>\max _{\boldsymbol{x} \in \mathcal{D}}\{b(\boldsymbol{x})\}-1$. The factor $1+\left(s / s_{1}\right)^{d}$ in the denominator ensures that the compliance relaxation function remains causal and that the wavefront remains traveling with the finite wave speed $c_{0}$ instead of the infinite wave speed that is implied by Eq. (18). Since $s_{1}$ is larger than the largest frequency of interest, the influence of the term will be practically negligible while theoretically avoiding the occurrence of a noncausal compliance relaxation function and associated problems.

After substitution of Eq. (19) into Eq. (16) and setting $s=j \omega$, it is easily shown that for $|\omega|<s_{1}$, the function $\gamma(x, j \omega)$ consists of an attenuation coefficient and a phase coefficient that can be approximated by

$$
\begin{aligned}
& \alpha(\boldsymbol{x}, \omega) \approx \alpha_{1}(\boldsymbol{x})|\omega|^{b(\boldsymbol{x})}, \\
& \beta(\boldsymbol{x}, \omega) \approx \frac{\omega}{c_{0}}+\alpha_{1}(\boldsymbol{x}) \tan (\pi b(\boldsymbol{x}) / 2) \omega|\omega|^{b(\boldsymbol{x})-1} .
\end{aligned}
$$

In the spatially homogeneous case, these coefficients correspond to those described by Szabo, ${ }^{32}$ and the propagation of a one way plane wave with angular frequency $\omega$ is just governed by these coefficients.

Equation (21) may be related to the phase speed $c(x, \omega)$ at angular frequency $\omega$ by using the relation $\beta(x, \omega)=\omega / c(x, \omega)$. This yields for $|\omega|<s_{1}$ the following dispersion equation:

$$
c(\boldsymbol{x}, \omega) \approx \frac{1}{c_{0}^{-1}+\alpha_{1}(\boldsymbol{x}) \tan (\pi b(\boldsymbol{x}) / 2)|\omega|^{b(\boldsymbol{x})-1}} .
$$

Spatially inhomogeneous attenuation is thus accompanied by a spatially inhomogeneous phase speed. Both types of inhomogeneities will influence the propagation and give rise to scattering of an acoustic wave. By modeling the attenuation via a compliance relaxation function, all relevant physical effects are automatically included via the applied attenuation contrast source.

From Eqs. (7) and (19) it follows that the function $\hat{A}(\boldsymbol{x}, s)$ in the attenuation contrast source in Eq. (11) is given by

$$
\begin{aligned}
\hat{A}(\boldsymbol{x}, s)= & \frac{2 c_{0} \alpha_{1}(\boldsymbol{x}) s^{b(\boldsymbol{x})-1}}{\cos (\pi b(\boldsymbol{x}) / 2)\left[1+\left(s / s_{1}\right)^{d}\right]} \\
& +\left(\frac{c_{0} \alpha_{1}(\boldsymbol{x}) s^{b(\boldsymbol{x})-1}}{\cos (\pi b(\boldsymbol{x}) / 2)\left[1+\left(s / s_{1}\right)^{d}\right]}\right)^{2} .
\end{aligned}
$$

Frequency domain results for $\hat{A}(\boldsymbol{x}, s)$ are obtained by taking $s=j 2 \pi f$, with $f$ being the temporal frequency. As an example, $|\hat{A}(f)|$ is plotted in Fig. 1 for liver, brain, blood, and water.

\section{SOLUTION METHOD}

Equations (13) and (14) describe a contrast source problem with known acoustic medium parameters and primary source, and an unknown acoustic pressure wave field. This problem is identified as a forward wave problem in the literature. In this Section, this problem is solved by employing the Neumann iterative solution.

\section{A. Neumann scheme}

Let the Green's function $G(x, t)$ be the solution of the wave equation

$$
\nabla^{2} G(\boldsymbol{x}, t)-\frac{1}{c_{0}^{2}} \partial_{t}^{2} G(\boldsymbol{x}, t)=-\delta(\boldsymbol{x}) \delta(t) .
$$

The Green's function is the field that is generated by the spatio-temporal impulsive source $\delta(x) \delta(t)$ in a homogeneous, linear, and lossless background medium. For the threedimensional case, the Green's function equals

$$
G(\boldsymbol{x}, t)=\frac{\delta\left(t-\|\boldsymbol{x}\| / c_{0}\right)}{4 \pi\|\boldsymbol{x}\|}
$$

with $\|x\|$ being the length of the position vector $x$. Supposing that the primary and contrast source in Eq. (13) are known, the solution of this equation reads

$$
p(\boldsymbol{x}, t)=G(\boldsymbol{x}, t) *_{\boldsymbol{x}, t}\left\{S_{\mathrm{pr}}(\boldsymbol{x}, t)+S_{\mathrm{cs}}[p(\boldsymbol{x}, t)]\right\} .
$$

Here, ${ }_{x, t}$ denotes a convolution over space and time. As the contrast source $S_{\mathrm{cs}}[p(x, t)]$ is a function of the unknown pressure field $p(x, t)$, Eq. (26) forms an integral equation. This equation may be solved via the Neumann iterative solution

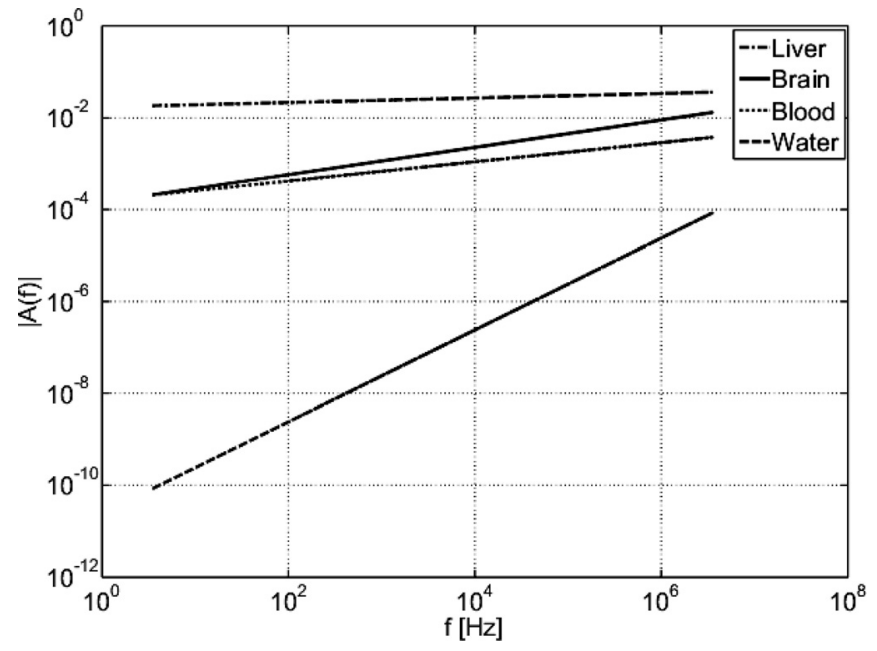

FIG. 1. The modulus of $\hat{A}(f)$ versus frequency, for liver, brain, blood, and water. 


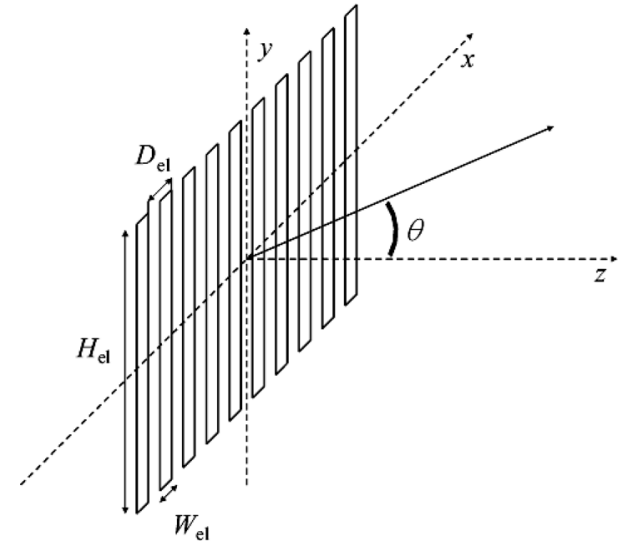

FIG. 2. The geometry of the phased array source. The number of elements is $N_{e l}=10$. The dimensions of each element are $W_{e l}=0.25 \mathrm{~mm}$ by $H_{e l}=1 \mathrm{~mm}$, and the pitch is $D_{e l}=1 \mathrm{~mm}$.

$$
\begin{aligned}
& p^{(n)}(\boldsymbol{x}, t)=0,(n<0), \\
& p^{(n)}(\boldsymbol{x}, t)=G(\boldsymbol{x}, t) *_{\boldsymbol{x}, t} S_{\mathrm{tot}}\left[p^{(n-1)}(\boldsymbol{x}, t)\right],(n \geq 0), \\
& S_{\mathrm{tot}}\left[p^{(n)}(\boldsymbol{x}, t)\right]=S_{\mathrm{pr}}(\boldsymbol{x}, t)+S_{\mathrm{cs}}\left[p^{(n)}(\boldsymbol{x}, t)\right],
\end{aligned}
$$

in which the total source term $S_{\text {tot }}\left[p^{(n)}(x, t)\right]$ represents the combined primary and contrast sources. This scheme defines the Neumann expansion ${ }^{33}$ of the acoustic pressure field. After discretization with respect to space and time, the following discrete Neumann scheme is obtained

$$
\begin{aligned}
& \mathbf{p}^{(n)}=0, \quad(n<0), \\
& \mathbf{p}^{(n)}=\mathbf{G}\left[\mathbf{S}_{\mathrm{tot}}\left[\mathbf{p}^{(n-1)}\right]\right], \quad(n \geq 0), \\
& \mathbf{S}_{\mathrm{tot}}\left[p^{(n)}\right]=\mathbf{S}_{\mathrm{pr}}+\mathbf{S}_{\mathrm{cs}}\left[\mathbf{p}^{(n)}\right],
\end{aligned}
$$

in which the vector $\mathbf{p}^{(n)}$ contains the $n$th order approximation of the acoustic pressure field at discrete grid points that span the spatiotemporal computational domain. Further, $\mathbf{G}$ is the operator that convolves the discrete Green's function with

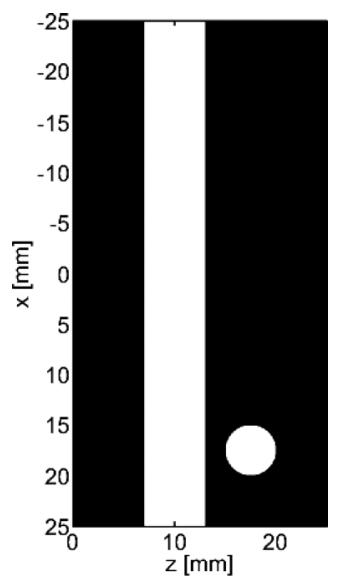

FIG. 3. Cross-section of configuration 2 along the plane $y=0$. Black indicates the lossless background medium, white indicates the lossy objects.

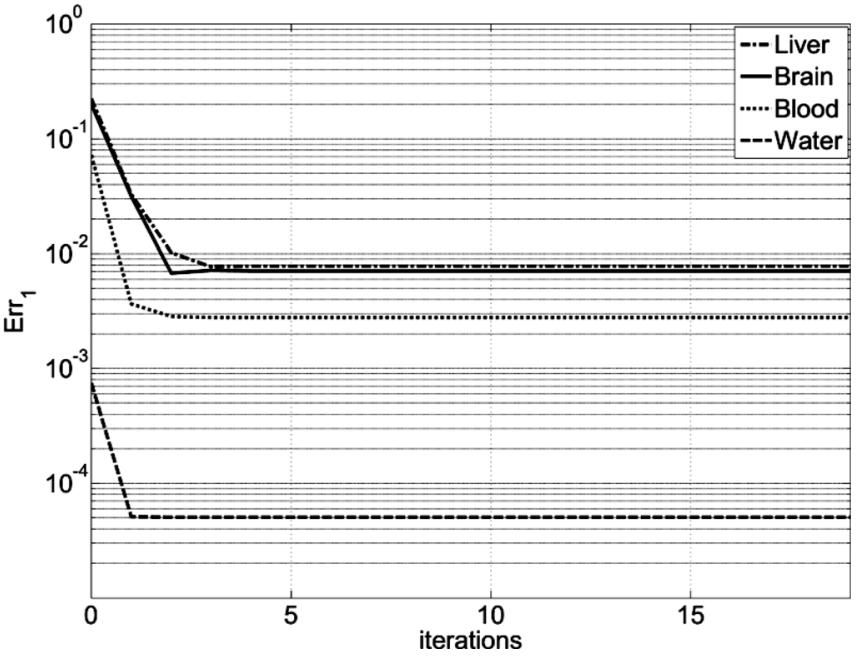

FIG. 4. Normalized error functional $\operatorname{Err}_{1}{ }^{(n)}$ for configuration 1 containing "linearized" $(\beta=0)$ liver, brain, blood, and water.

the discrete total source term $S_{\text {tot }}\left[\mathbf{p}^{(n)}\right]$, which is the discrete version of $S_{\text {tot }}\left[p^{(n)}(x, t)\right]$.

For the actual implementation of the scheme, the filtered convolution method ${ }^{10,13}$ is employed. This method uses filtering of the Green's function and the source terms to avoid singularities and aliasing effects, and it allows for a course discretization of only two points per wavelength and per period at the highest frequency of interest. After discretization, the spatiotemporal convolution is performed with the aid of an efficient FFT technique.

\section{B. Convergence}

The convergence of the Neumann scheme is known to depend on the magnitude and the spatial extent of the contrast source. For a theoretical analysis of the convergence of the scheme, the reader is referred to the literature. ${ }^{34}$ To verify the numerical convergence of the Neumann scheme toward the exact solution, a normalized error functional $\operatorname{Err}_{1}^{(n)}$ is introduced as

$$
\operatorname{Err}_{1}^{(n)}=\frac{\left\|\mathbf{p}^{\mathrm{ex}}-\mathbf{p}^{(n)}\right\|}{\left\|\mathbf{p}^{\mathrm{ex}}\right\|}
$$

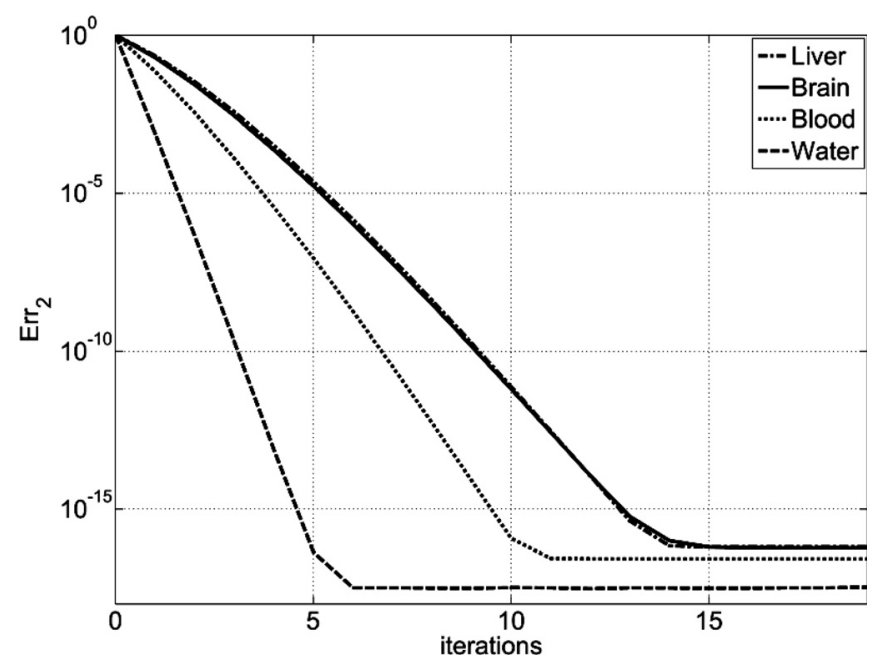

FIG. 5. Normalized error functional $\operatorname{Err}_{2}{ }^{(n)}$ for configuration 1 containing "linearized" $(\beta=0)$ liver, brain, blood, and water. 
in which $\mathbf{p}^{\mathrm{ex}}$ equals the exact solution as obtained from a benchmarking program. When $\operatorname{Err}_{1}^{(n)}$ decreases toward zero for increasing $n$, the obtained solution converges toward the exact solution. To test the numerical convergence of the Neumann scheme toward a steady solution, a second normalized error functional $\operatorname{Err}_{2}^{(n)}$ is defined as

$$
\operatorname{Err}_{2}^{(n)}=\frac{\left\|\mathbf{p}^{(n-1)}-\mathbf{p}^{(n)}\right\|}{\left\|\mathbf{p}^{(0)}\right\|} .
$$

When $\operatorname{Err}_{2}^{(n)}$ decreases toward zero for increasing $n$, the obtained solution converges toward a steady solution. The numerical convergence of the scheme is demonstrated in Sec. IV B.

\section{RESULTS}

\section{A. Configurations}

Two configurations are used to demonstrate the numerical performance of the presented method. In both configura-

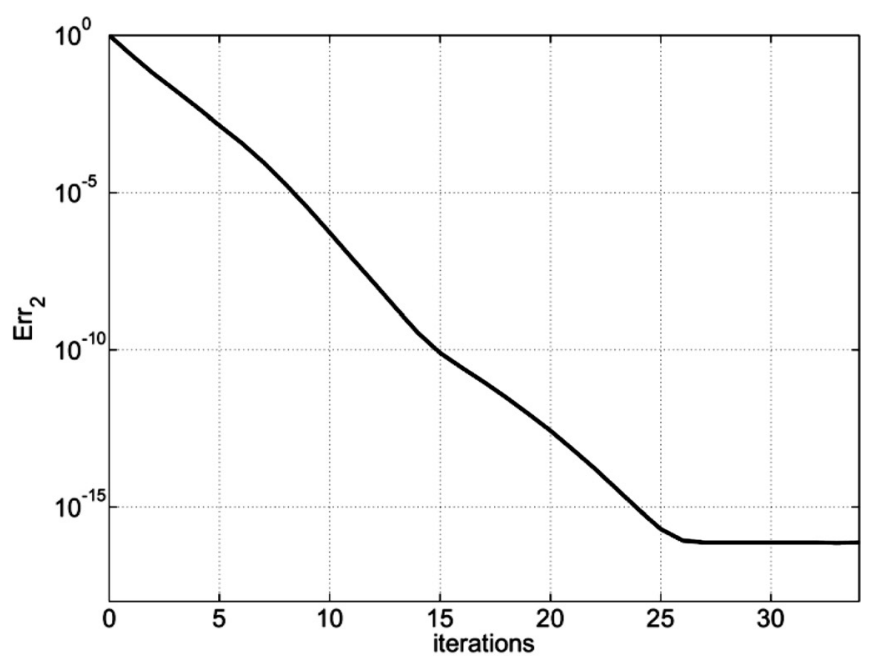

(a)

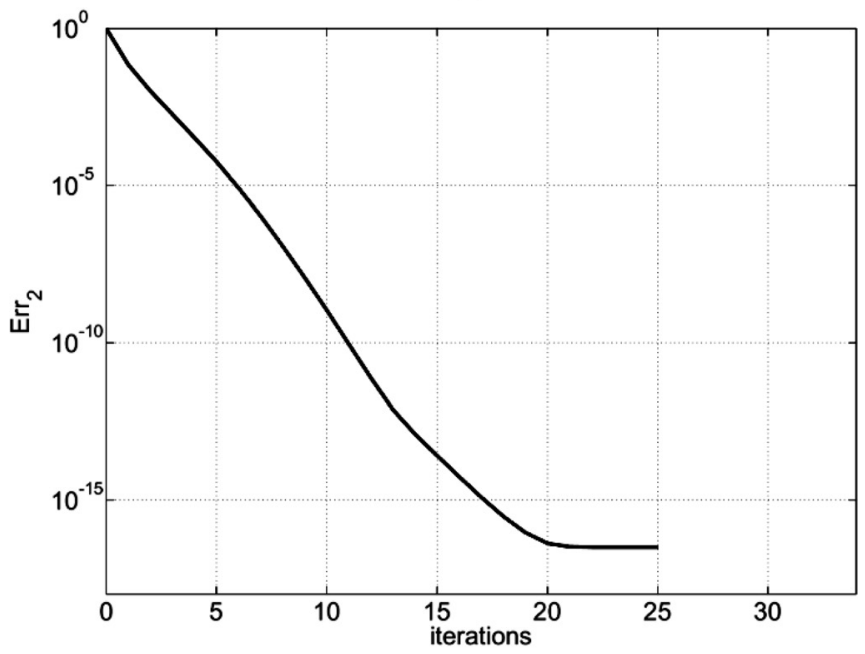

(b)

FIG. 6. The normalized error functional $\operatorname{Err}_{2}^{(\mathrm{n})}$; (a) for configuration 1 (homogeneous lossy liver), (b) for configuration 2 (lossy liver objects in a lossless liver background). tions, the source is a linear phased array. The origin of the coordinate system is located at the center of the transducer. A graphical representation of the source is given in Fig. 2. The array consists of $N_{e l}=10$ elements. The dimensions of the elements are $W_{e l}=0.25 \mathrm{~mm}$ by $H_{e l}=10 \mathrm{~mm}$, and the pitch is $D_{e l}=1 \mathrm{~mm}$. The elements are excited with a pulse consisting of an harmonic signal with a frequency $f_{0}=1$ $\mathrm{MHz}$ that is amplitude modulated by a Gaussian pulse with a width $t_{w}=3 / f_{0}$. This envelope contains about six cycles of the harmonic signal. ${ }^{11}$ The peak pressure at the surface of a transducer element is $P_{0}=0.5 \mathrm{MPa}$. No focusing is applied.

Configuration 1 may contain different types of homogeneous lossy media. No beam steering is applied, and the dimensions of the spatial computational domain $\mathcal{D}$ are $(x \times y \times z)=(25 \mathrm{~mm} \times 20 \mathrm{~mm} \times 50 \mathrm{~mm})$. Configuration 2 contains lossy objects embedded in a corresponding lossless background medium. The transducer beam is steered over an angle $\theta=45$, and the spatial computational domain $\mathcal{D}$

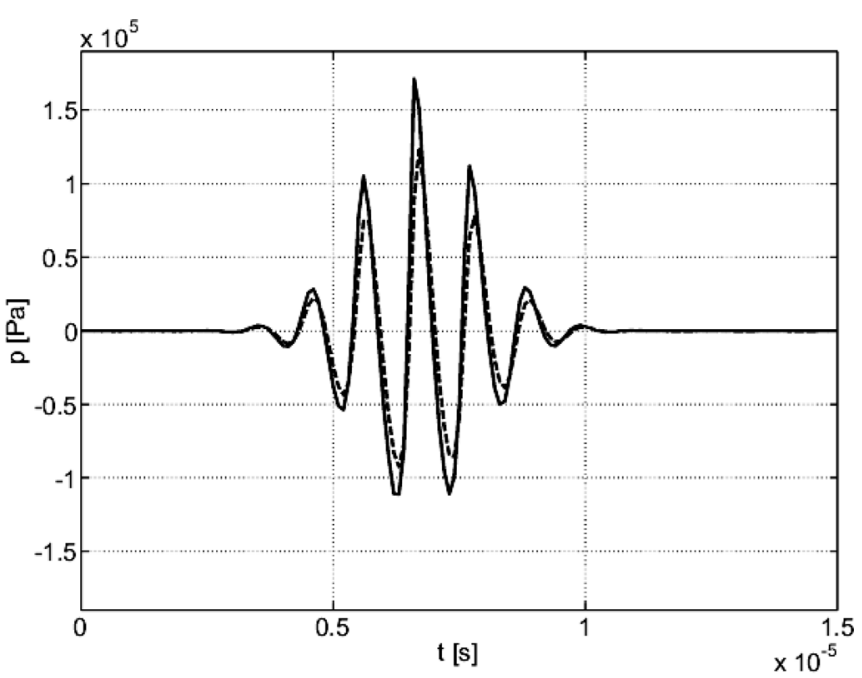

(a)

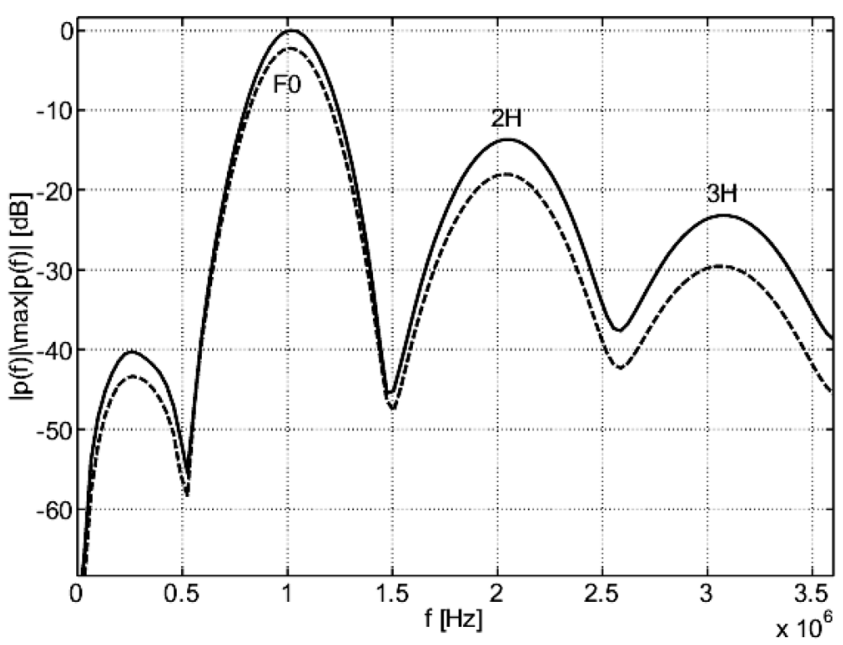

(b)

FIG. 7. The nonlinear pressure wave in configuration 1 with lossless (solid line) and lossy (dashed line) liver; (a) time domain pulse, (b) normalized frequency domain spectra. The observation point is $(x, y, z)=(0 \mathrm{~mm}, 0 \mathrm{~mm}$, $50 \mathrm{~mm})$. The graphs apply to the fundamental $(\mathrm{F} 0)$, second harmonic $(2 \mathrm{H})$, and third harmonic $(3 \mathrm{H})$ components. 


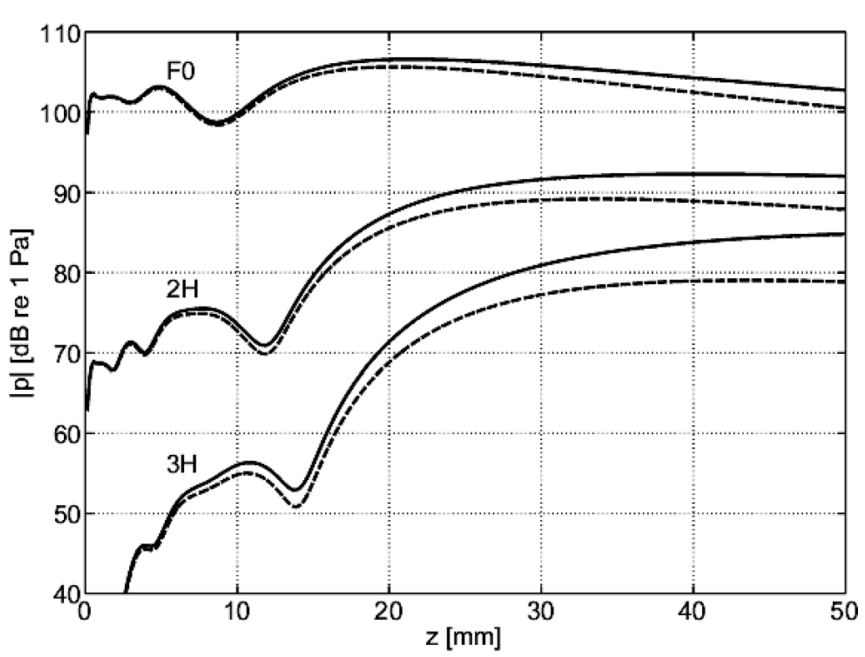

(a)

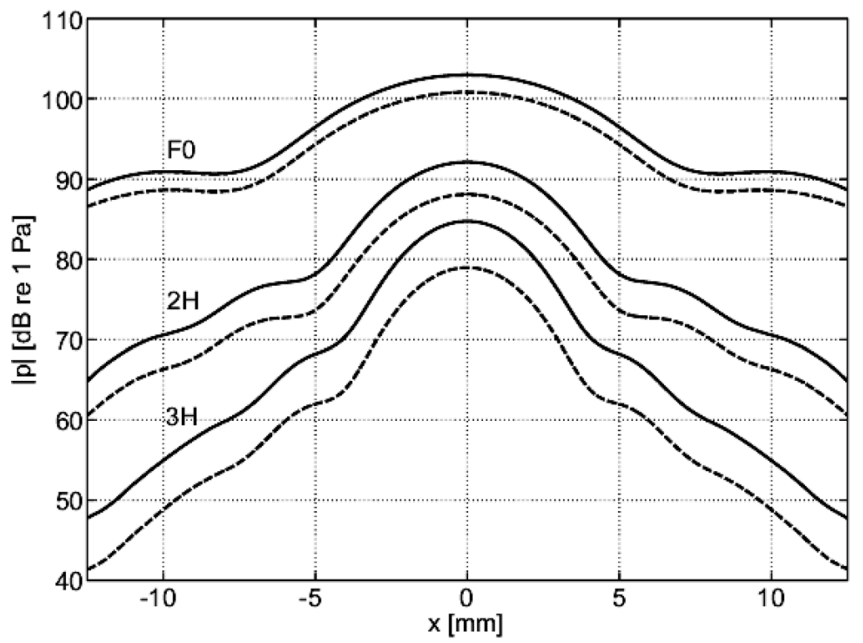

(b)

FIG. 8. The nonlinear pressure field in configuration 1 with lossless (solid line) and lossy (dashed line) liver; (a) axial profile evaluated along the $z$ axis, (b) lateral profile evaluated along the line $(y, z)=(0 \mathrm{~mm}, 50 \mathrm{~mm})$. The graphs apply to the fundamental (F0), second harmonic $(2 \mathrm{H})$, and third harmonic $(3 \mathrm{H})$ components.

measures $(x \times y \times z)=(50 \mathrm{~mm} \times 20 \mathrm{~mm} \times 25 \mathrm{~mm})$. For configuration 2 , a cross-section of the spatial domain $\mathcal{D}$ along the plane $y=0$ is shown in Fig. 3.

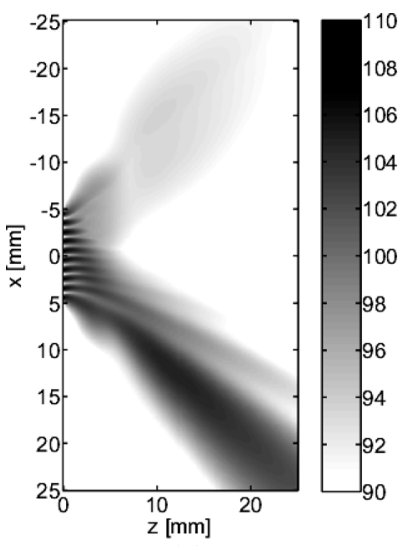

(a)

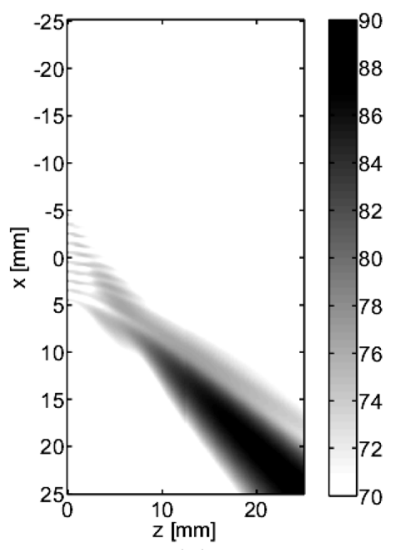

(b)

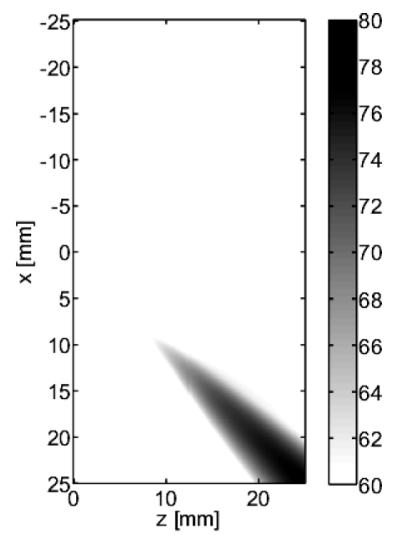

(c)
FIG. 9. Two-dimensional spectral profiles of the pressure wave field in configuration 2 with lossless liver and lossy inhomogeneities; (a) fundamental, (b) second harmonic, (c) third harmonic. The observation plane is $y=0 \mathrm{~mm}$. The pressure values are expressed in $\mathrm{dB}$ re $1 \mathrm{~Pa}$. 
containing lossy liver objects in a lossless liver background. The results are presented in Fig. 6. For configuration 1, the flattening of $\operatorname{Err}_{2}^{(n)}$ is reached within 26 iterations, whereas the inhomogeneous configuration 2 requires only 19 iterations. The difference may likely be caused by the fact that, unlike configuration 1, configuration 2 has an attenuation contrast source that is zero outside the objects, resulting in a smaller volume of the total contrast source.

\section{In silico experiments}

A numerical example of the nonlinear propagation of the acoustic pressure wave is presented in Fig. 7. This figure applies to configuration 1 with both lossless (indicated as "background" in Table I) and lossy liver. Figure 7(a) shows the time domain pressure pulse in the point $(x, y, z)=(0 \mathrm{~mm}$, $0 \mathrm{~mm}, 50 \mathrm{~mm}$ ). The normalized frequency spectra of this pulse are shown in Fig. 7(b), where the fundamental (F0), the second harmonic $(2 \mathrm{H})$, and the third harmonic $(3 \mathrm{H})$ component of the pressure wave field are clearly visible. Normalization is performed with respect to the maximum absolute value of the lossless spectrum. Both figures clearly show a reduction of the amplitude of the pressure wave when attenuation is taken into account. The frequency dependent nature of the attenuation is visible in Fig. 7(b), in which an increase in attenuation is observed for increasing frequency. This is in agreement with the behavior of $\hat{A}(f)$ for liver, as shown in Fig. 1.

Figure 8 shows the axial and lateral spectral profiles of the pressure field that is generated in configuration 1 with lossless and lossy liver. The axial profile in Fig. 8(a) is evaluated along the $z$ axis, and the lateral profile in Fig. 8(b) is evaluated along the line $(y, z)=(0 \mathrm{~mm}, 50 \mathrm{~mm})$. In both panels, the increase of attenuation for the higher harmonics is clearly visible. The cumulative nature of the nonlinear effects may be observed in Fig. 8(a), which shows the growth of the amplitudes of the harmonics with depth.

In Fig. 8(b), two effects are recognizable that are connected to nonlinear propagation and that are utilized in

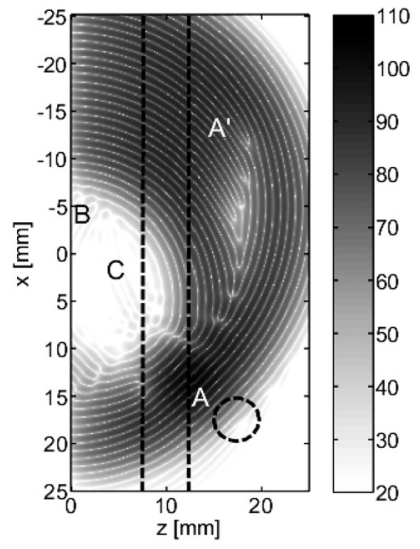

(a)

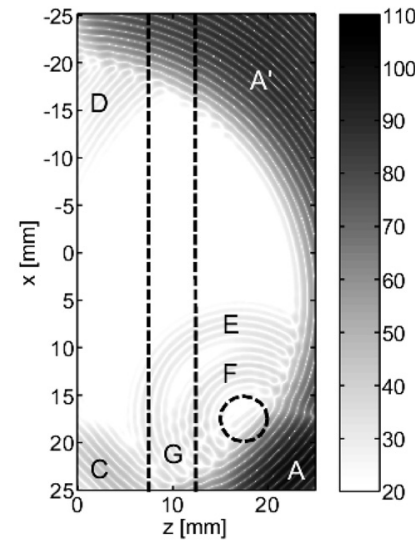

(b)
FIG. 10. Snapshots of the propagating pressure pulse in configuration 2 with lossless liver (background) and lossy inhomogeneities (dashed objects); (a) early time, (b) late time. The observation plane is $y=0 \mathrm{~mm}$. The labels refer to the phenomena described in the text, and in Table II. The pressure values are expressed in $\mathrm{dB}$ re $1 \mathrm{~Pa}$.

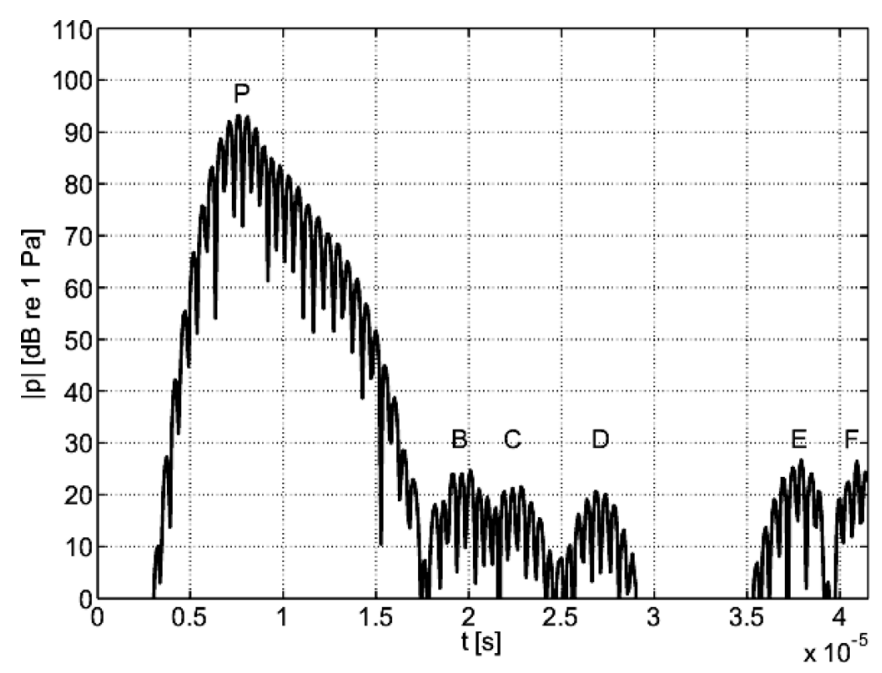

FIG. 11. Pressure versus time at the origin of the coordinate system in configuration 2 with lossless liver and lossy inhomogeneities. The observation point is $(x, y, z)=(0 \mathrm{~mm}, 0 \mathrm{~mm}, 0 \mathrm{~mm})$. The labels refer to the phenomena described in the text, and in Table II.

medical applications to improve the quality of ultrasound images. First, if the ratio between the amplitude of the main and side lobes is considered, a reduction of this ratio is observed for increasing harmonics. Second, for increasing harmonics, the main beam width decreases. In particular, the $-3 \mathrm{~dB}$ beamwidth is $6.9 \mathrm{~mm}$ for the fundamental, $4.3 \mathrm{~mm}$ for the second harmonic, and $3.4 \mathrm{~mm}$ for the third harmonic.

Figure 9 shows the two-dimensional spectral profiles of the nonlinear pressure field in the plane $y=0 \mathrm{~mm}$ for configuration 2 with lossy (objects) and lossless liver (background).

For increasing harmonics, the decreasing size of the focal area and the grating lobes, and the clutter (near field) reduction are clearly visible in these figures. Note that grating lobes and related effects can only be modeled accurately when it is possible to compute pressure wave fields over a wide angle. The INCS method allows to accurately do this since no a priori assumption on the directivity of the wave field is employed. ${ }^{10,11}$

Two snapshots of the pressure wave propagating in configuration 2 with lossy (objects) and lossless liver (background) are shown in Fig. 10. The fields are presented for the plane $y=0 \mathrm{~mm}$. In Fig. 11, pressure versus time at the origin of the coordinate system is shown. In both 10 and 11, the characters $\mathrm{A}$ to $\mathrm{P}$ are used to mark specific phenomena, see Table II. The generated pressure wave field $\mathrm{P}$ originates from the transducer and travels through the lossless background.

TABLE II. Acoustical phenomena present in Figs. 10 and 11.

\begin{tabular}{lc}
\hline \hline Character & Acoustical phenomenon \\
\hline A & Main beam \\
A & Grating lobe \\
B & Reflection from the front of the slab \\
C-D & Reflection from the back of the slab \\
E & Reflection from the front of the cylinder \\
F & Reflection from the back of the cylinder \\
P & Direct field of the transducer \\
\hline \hline
\end{tabular}


Part of the pressure wave field contributes to the formation of the main beam $\mathrm{A}$ and another part to the formations of the grating lobe A'. Furthermore, when the pressure wave field encounters an interface between the background and the inhomogeneities, part of the field propagates through and part is backscattered. In specific, B marks the backscattered pressure wave field caused by the front of the lossy slab, C and D mark the backscattered field caused by the back of the lossy slab and $\mathrm{E}$ and $\mathrm{F}$ mark, respectively, the backscattered field caused by the front and the back of the cylinder.

\section{CONCLUSIONS}

In this paper, a novel method has been presented to accurately solve the wave equation for an acoustic nonlinear medium with spatially inhomogeneous attenuation. This wave equation is obtained by extending the Westervelt equation with a causal compliance relaxation function to incorporate attenuation. A specific form of the compliance relaxation function has been proposed to provide the frequency power law losses that are usually encountered in biological tissue. Spatially dependent attenuation has been introduced by taking a spatially dependent relaxation function. The approach automatically provides the corresponding spatially dependent phase speed. The spatial dependence of both the attenuation and the phase speed gives rise to scattering of an acoustic wave. The resulting wave equation leads to the formulation of a contrast source problem that has been solved iteratively using a Neumann scheme. Since the presented method is an extension of the lossless INCS method, it is able to accurately solve the full-wave equation for the weakly to moderately nonlinear, wide-angle, pulsed acoustic wave field in a large, three-dimensional domain. Convergence of the method has been investigated and results for homogeneous, lossy, linear media show full agreement with the exact results. Moreover numerical results have been generated to demonstrate the influence of the frequency-dependent attenuation on a pulsed beam in a nonlinear medium with homogeneous attenuation. For a nonlinear medium with spatially inhomogeneous attenuation, the scattering from attenuating objects has been shown as well.

${ }^{1}$ M. A. Averkiou, D. N. Roundhill, and J. E. Powers, "A new imaging technique based on the nonlinear properties of tissues," in Proceedings of the 1997 IEEE Ultrasonics (1997), pp. 1561-1566.

${ }^{2}$ I. M. Hallaj and R. O. Cleveland, "FDTD simulation of finite-amplitude pressure and temperature fields for biomedical ultrasound," J. Acoust. Soc. Am. 105, L7-L12 (1999).

${ }^{3}$ B. Ward, A. C. Baker, and V. F. Humphrey, "Nonlinear propagation applied to the improvement of resolution in diagnostic medical ultrasound equipment," J. Acoust. Soc. Am. 101, 143-154 (1999).

${ }^{4}$ F. Tranquart, N. Grenier, V. Eder, and L. Pourcelot, "Clinical use of ultrasound tissue harmonic imaging," Ultrasound Med. Biol. 25, 889-894 (1999). ${ }^{5} \mathrm{~A}$. Bouakaz and N. de Jong, "Native tissue imaging at superharmonic frequencies," IEEE Trans. Ultrason. Ferroelectr. Freq. Control 50, 496506 (2003).

${ }^{6}$ A. Bouakaz, E. Merks, C. T. Lancee, and N. Bom, "Non invasive bladder volume measurements based on nonlinear wave distortion," Ultrasound Med. Biol. 30, 469-476 (2004).

${ }^{7} \mathrm{P}$. N. T. Wells, "Absorption and dispersion of ultrasound in biological tissue," Ultrasound Med. Biol. 1, 369-376 (1985).

${ }^{8}$ F. A. Duck, Physical Properties of Tissue (Academic Press, London, 1990), pp. 99-123.
${ }^{9}$ E. A. Filonenko and V. A. Khokhlova, "Effect of acoustic nonlinearity on heating of biological tissue induced by high intensity focused ultrasound," Acoust. Phys. 47, 468-475 (2001).

${ }^{10}$ J. Huijssen, "Modeling of nonlinear medical diagnostic ultrasound," Ph.D. thesis, Delft University of Technology, 35-120 and 171-186 (2008), available at http://repository.tudelft.nl (Last viewed June 24, 2010).

${ }^{11}$ J. Huijssen and M. D. Verweij, "An iterative method for the computation of nonlinear, wide-angle, pulsed acoustic fields of medical diagnostic transducers," J. Acoust. Soc. Am. 127, 33-44 (2010).

${ }^{12}$ M. F. Hamilton and C. L. Morfey, "Model equations," in Nonlinear Acoustics, edited by M. F. Hamilton and D. T. Blackstock (Academic Press, San Diego, 1998), pp. 54-56.

${ }^{13}$ M. D. Verweij and J. Huijssen, "A filtered convolution method for the computation of acoustic wave fields in very large spatiotemporal domains," J. Acoust. Soc. Am. 125, 1868-1878 (2009).

${ }^{14}$ J. Huijssen, M. D. Verweij, and N. de Jong, "Green's function method for modeling nonlinear three dimensional pulsed acoustic fields in diagnostic ultrasound including tissue-like attenuation," in Proceedings of the 2008 IEEE Ultrasonics (2008), pp. 375-378.

${ }^{15}$ J. F. Bakker, M. M. Paulides, I.-M. Obdeijn, G. C. van Rhoon, and K. W. A. van Dongen, "An ultrasound cylindrical phased-array for deep heating in the breast: Theoretical design using heterogeneous models," Phys. Med. Biol. 54, 3201-3215 (2009).

${ }^{16}$ M. D. Verweij, "Modeling space-time domain acoustic wave fields in media with attenuation: The symbolic manipulation approach," J. Acoust. Soc. Am. 97, 831-43 (1995).

${ }^{17}$ M. D. Verweij, "Transient acoustic wave fields in continuously layered media with depth-dependent attenuation: An analysis based on higherorder asymptotics," J. Acoust. Soc. Am. 101, 1808-1820 (1997).

${ }^{18}$ L. Demi, M. D. Verweij, J. Huijssen, N. de Jong, and K. W. A. van Dongen, "Attenuation of ultrasound pressure fields described via a contrast source formulation," in Proceedings of the 2009 IEEE Ultrasonics (2009), pp. $1590-1593$.

${ }^{19}$ R. O. Cleveland, M. F. Hamilton, and D. T. Blackstock, "Time-domain modeling of finite-amplitude sound in relaxing fluids," J. Acoust. Soc. Am. 99, 3312-3318 (1996).

${ }^{20}$ G. F. Pinton, J. Dahl, S. Rosenzweig, and G. E. Trahey, "A heterogeneous nonlinear attenuating wave model of ultrasound," IEEE Trans. Ultrason. Ferroelectr. Freq. Control 56, 474-488 (2009).

${ }^{21}$ D. T. Blackstock, "Generalized Burgers equation for plane waves," J. Acoust. Soc. Am. 77, 2050-2053 (1985).

${ }^{22}$ V. A. Khokhlova, A. E. Ponomarev, M. A. Averkiou, and L. A. Crum, "Nonlinear pulsed ultrasound beams radiated by rectangular focused diagnostic transducers," Acoust. Phys. 52, 481-489 (2006).

${ }^{23}$ S. I. Aanonsen, T. Barkve, J. Naze Tjøtta, and S. Tjøtta, "Distortion and harmonic generation in the nearfield of a finite amplitude sound beam," J. Acoust. Soc. Am. 74, 749-768 (1984)

${ }^{24}$ D. V. Widder, The Laplace Transform (Princeton University Press, New York, 1946), pp. 61-63.

${ }^{25}$ G. Doetsch, Handbuch der Laplace Transformation, Vol. 1 (Handbook of the Laplace Transformation) (Birkhäuser, Basel, 1950), p. 73.

${ }^{26}$ J. P. Schouten, Operatorenrechnung mit Anwendungen auf Technische Probleme (Operational calculus with applications to technical problems) (Springer-Verlag, Berlin, 1961), pp. 101-103.

${ }^{27}$ R. E. A. C. Paley and N. Wiener, "Fourier transforms in the complex domain," Am. Math. Soc. Colloq. Pub. 19, 116-117 (1934).

${ }^{28}$ A. T. de Hoop, Handbook of Radiation and Scattering of Waves (Academic Press, London, (1995), pp. 71-75 and 1065-1070, available at http://www.atdehoop.com (Last viewed June 24, 2010).

${ }^{29}$ J. C. Bamber, "Ultrasonic properties of tissues," in Ultrasound in Medicine, edited by F. A. Duck, A. C. Baker, and H. C. Starritt (Institute of Physics, Bristol, 1998), pp. 73-76.

${ }^{30}$ T. L. Szabo, Diagnostic Ultrasound Imaging (Elsevier, Amsterdam, 2004), p. 535.

${ }^{31}$ R. S. C. Cobbold, Foundations of Biomedical Ultrasound (Oxford University Press, Oxford, 2007), pp. 72-81.

${ }^{32}$ T. L. Szabo, "Causal theories and data for acoustic attenuation obeying a frequency power law," J. Acoust. Soc. Am. 97, 14-24 (1995).

${ }^{33}$ J. T. Fokkema and P. M. van den Berg, Seismic Applications of Acoustic Reciprocity (Elsevier, Amsterdam, 1993), pp. 54-55.

${ }^{34} \mathrm{~A}$. T. de Hoop, "Convergence criterion for the time-domain iterative Born approximation to scattering by an inhomogeneous, dispersive object," J. Opt. Soc. Am. A 8, 1256-1260 (1991). 